\title{
Attenuated p53 activation in tumour-associated stromal cells accompanies decreased sensitivity to etoposide and vincristine
}

\author{
AC Dudley', S-C Shih ${ }^{2}$, AR Cliffe ${ }^{3}, \mathrm{~K} \mathrm{Hida}^{4}$ and M Klagsbrun*,1,5 \\ 'Vascular Biology Program, Department of Surgery, Children's Hospital and Harvard Medical School, Boston, MA, USA; ${ }^{2}$ Department of Pathology, Beth \\ Israel Deaconess Medical Center and Harvard Medical School, Boston, MA, USA; ${ }^{3}$ Department of Microbiology and Molecular Genetics, Harvard Medical \\ School, Boston, MA, USA; ${ }^{4}$ Hokkaido University, Grad. School of Dental Medicine and Oral Pathology, Sapporo, Japan; ${ }^{5}$ Department of Pathology, \\ Children's Hospital and Harvard Medical School, Boston, MA, USA
}

Alterations in the tumour suppressor p53 have been reported in tumour-associated stromal cells; however, the consequence of these alterations has not been elucidated. We investigated p53 status and responses to p53-activating drugs using tumour-associated stromal cells from A375 melanoma and PC3 prostate carcinoma xenografts, and a spontaneous prostate tumour model (TRAMP). p53 accumulation after treatment with different p53-activating drugs was diminished in tumour-associated stromal cells compared to normal stromal cells. Tumour-associated stromal cells were also less sensitive to p53-activating drugs - this effect could be reproduced in normal stromal cells by $\mathrm{p} 53$ knockdown. Unlike normal stromal cells, tumour stromal cells failed to arrest in $\mathrm{G}_{2}$ after etoposide treatment, failed to upregulate p53-inducible genes, and failed to undergo apoptosis after treatment with vincristine. The lower levels of p53 in tumour stromal cells accompanied abnormal karyotypes and multiple centrosomes. Impaired p53 function in tumour stroma might be related to genomic instability and could enable stromal cell survival in the destabilising tumour microenvironment.

British Journal of Cancer (2008) 99, I I8- 125. doi:10.1038/sj.bjc.6604465 www.bjcancer.com

Published online I July 2008

(c) 2008 Cancer Research UK

Keywords: tumour; tumour microenvironment; tumour stroma; p53; drug resistance

Both cancer and healing wounds are characterised by increased proliferation and infiltration of endothelial cells, inflammatory cells, and fibroblasts (Dvorak, 1986). These infiltrating stromal cells (SC) may by tissue-resident and/or bone marrow-derived and together constitute the stromal microenvironment. The function of SC in tumours and physiological wound healing is to secrete matrix proteins and factors involved in tissue remodelling and to secrete chemotactic factors for inflammatory cells and endothelial cells. Thus, both tissue injury and cancer resemble an 'activated' state whereby the host's response is designed to heal the affected tissue (Coussens and Werb, 2002).

The tumour microenvironment consists of tumour cells, SC, and the matrix proteins, growth factors, and cytokines they produce (Liotta and Kohn, 2001). Fibroblasts comprise the majority of the tumour stroma, and their role in tumours is long-recognised (Seemayer et al, 1979). For example, fibroblasts are responsible for the synthesis of fibronectin and types I, III, and V collagens that make up basement membranes; they also secrete factors such as TGF- $\beta$ that support nonautonomous tumour epithelial growth (Tlsty, 2001; Bhowmick et al, 2004). Functionally and phenotypically distinct myofibroblasts are also observed in most tumours

*Correspondence: Dr M Klagsbrun, Vascular Biology Program, Department of Surgery, Children's Hospital and Harvard Medical School, 300 Longwood Avenue, Boston, MA 021 I 5, USA; E-mail: michael.klagsbrun@childrens.harvard.edu

Received 2 May 2008; accepted I5 May 2008; published online I July 2008
(Sappino et al, 1988). Myofibroblasts express $\alpha$-smooth muscle actin ( $\alpha$-SMA), which enhances their contractility and motility (Hinz et al, 2007), and they overexpress stromal-derived factor, which mobilises bone marrow-derived endothelial progenitor cells (Orimo et al, 2005).

Owing to genomic instability, cancer cells are typically mutable and develop drug resistance; thus, targeting SC in the tumour microenvironment may be a viable approach for helping to eliminate solid tumours (e.g., antiangiogenesis). However, it has recently become clear that like tumour cells, tumour-associated SC may be characterised by genomic instability and p53 mutations (Kurose et al, 2002; Hill et al, 2005). For example, numerous genetic amplifications and deletions were detected in murine stromal DNA isolated from implanted tumour xenografts (Pelham et al, 2006). Furthermore, cytogenetic (Moinfar et al, 2000; Allinen et al, 2004; Fukino et al, 2004; Hida et al, 2004) and epigenetic (Kaplan et al, 2005) alterations have been described in tumour stroma and in tissue adjacent to carcinoma (Deng et al, 1994). The possibility that cytogenetic alterations in tumour SC including p53 mutations might accompany changes in $\mathrm{p} 53$ function has not been addressed.

In cancer cells, the principal cause of resistance to chemotherapeutic drugs is chromosomal instability accompanied by deletion of or mutations in p53; however, both drug resistance and increased sensitivity in a p53 null background have been noted (Bunz et al, 1999; Burdelya et al, 2006). p53 is mutated or lost in about half of all cancers probably as a consequence of selection pressure for p53 mutations, which enable tumour cell survival (Gorgoulis et al, 2005). The importance of functional p53 in tumours has been formally proven by reintroduction of wild-type 
p53 in tumour cells in vivo (Martins et al, 2006; Ventura et al, 2007). However, it has been noted that re-imposition of $\mathrm{p} 53$ function was quickly mitigated by p53 inactivation and the emergence of p53-resistant tumours (Martins et al, 2006). Despite these detailed studies investigating p53 function in tumour cells, no studies to date have examined the relationship between p53 status and sensitivity to cytotoxic DNA-damaging agents in tumour-associated SC.

In this report, we show that tumour SC, in contrast to normal SC, fail to undergo growth arrest and apoptosis after treatment with etoposide or vincristine, respectively. Tumour SC show diminished p53 expression, and p53 fails to or only marginally accumulates after treatment with p53-activating stimuli. Tumour SC also have multiple centrosomes and are aneuploid - both of which are readouts of abnormal p53 function. Together, these results indicate that, similar to tumour cells, alterations in p53 function and decreased sensitivity to commonly used p53-activating chemotherapeutic drugs are features of tumour-associated SC.

\section{MATERIALS AND METHODS}

\section{Cell lines and media}

All primary cells were cultured in EGM2-MV medium (Cambrex Bioscience, Rockland, ME, USA) and were maintained in an atmosphere of $5 \% \mathrm{CO}_{2}$ at $37^{\circ} \mathrm{C}$. PC3MLN4 prostate carcinoma cells were grown in HAMS with 10\% FBS, and A375SM melanoma cells were grown in MEM with $10 \%$ FBS. Tumour cells were grown under $10 \% \mathrm{CO}_{2}$. Normal and tumour SC were obtained from isolated endothelial cultures overtaken by rapidly growing fibroblast-like cells. This was a common occurrence in our hands, and was unavoidable without strict monitoring of the pure endothelial cultures (Hida et al, 2004).

\section{Mice}

All animal procedures were performed in compliance with Boston Children's Hospital Guidelines and were approved by the Institutional Animal Use and Care Committee. The procedures for cell isolation for A375 tumour xenografts were previously described (Hida et al, 2004). For the PC3MLN4 prostate carcinoma, one million cells were injected subcutaneously into the dorsal lateral flank of 8-week-old nu/nu mice. When tumours reached $1 \mathrm{~cm}^{3}$ (approximately $4-6$ weeks postimplantation), tumours were harvested as described by Hida et al (2004). Tumours from five mice were combined for the cell isolation. TRAMP mice were genotyped at 4 weeks of age (Transnetyx, Cordova, TN, USA) and tumours were harvested when mice reached 20-22 weeks.

\section{Antibodies and reagents}

The mouse monoclonal p53 and rabbit polyclonal pSER 15 and pSER20 p53 antibodies were purchased from Cell Signaling Technologies (Danvers, MA, USA). $\beta$-actin, caldesmon, $\alpha$-SMA, and fibroblast-specific protein-1 (FSP-1) antibodies were from Sigma-Aldrich (St Louis, MO, USA). The CD105 antibody was from BD Pharmingen (San Jose, CA, USA). Rabbit polyclonal pericentrin antibody was from Covance (Berkeley, CA, USA). Etoposide and vincristine were dissolved in DMSO and were purchased from Sigma-Aldrich.

\section{Drug treatment}

To analyse p53 expression, cells were seeded at a density of 200000 cells per $10 \mathrm{~cm}^{2}$ and left overnight. The next day, the indicated drug was added, and the cells were incubated between 8 and $24 \mathrm{~h}$. Cell lysates were prepared in RIPA buffer and subjected to western blotting according to standard methods. Nuclear fractions were prepared using the NE-PER kit according to the manufacturer (Pierce, Rockford, IL, USA). For viability studies, cells were seeded at 2500 cells per well in a 96-well plate. The next day, the indicated concentration of each drug was added, and the cells were incubated for an additional $72 \mathrm{~h}$. Cell counts were determined by dispersing the cells in trypsin and counted using a Coulter counter (Beckman Coulter, Fullerton, CA, USA; Model Z1).

\section{Fluorescence-activated cell sorting}

Fluorescence-activated cell sorting (FACS) for cell characterisation was carried out on live cells used between 6 and 10 passages. Propidium iodide and annexin V staining were done according to the manufacturer's instructions (BD Pharmingen). Cells were analysed on a BD FACSCalibur System.

\section{Immunofluorescence}

Cells were seeded at a density of 10000 cells per well in gelatincoated eight-well chamber slides. Confluent cells were washed twice with PBS and then fixed with ice-cold 100\% methanol at $-20^{\circ} \mathrm{C}$ for $20 \mathrm{~min}$. The fixed cells were rinsed briefly with PBS and then blocked for 1 hour at room temperature with PBS containing $5 \%$ BSA. After blocking, antibodies were added overnight at $4{ }^{\circ} \mathrm{C}$ in a humidified chamber. The next day, cells were rinsed with PBS and then blocked again for $30 \mathrm{~min}$ at room temperature. Secondary antibodies were added and the cells were incubated an additional hour at room temperature protected from light. Finally, the cells were washed with PBS and then mounted using Gel Mount (Biomeda, Foster City, CA, USA) containing $0.4 \mu \mathrm{g} \mathrm{ml}^{-1}$ 4',6-diamidino-2-phenylindole.

\section{p53 sequencing}

cDNA was prepared from normal and tumour SC by reverse transcription. Primers specific for the mouse p53-coding regions

A
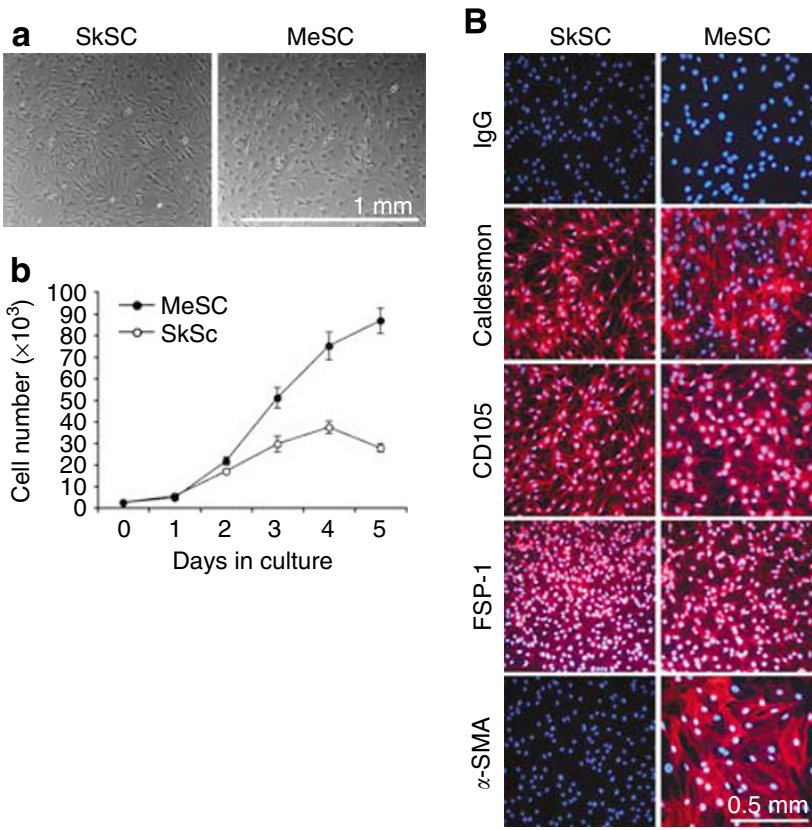

Figure I Stromal cell characterisation. (A) Morphology of SC from normal mouse skin or subcutaneous xenografts of A375 melanoma (a) and comparison of their growth properties in culture (b). (B) By immunofluorescence, SC stained uniformly positive for caldesmon, CDI05, and FSP-I. $\alpha$-SMA was expressed only in the tumour SC, indicative of a myofibroblast-like phenotype. 
A
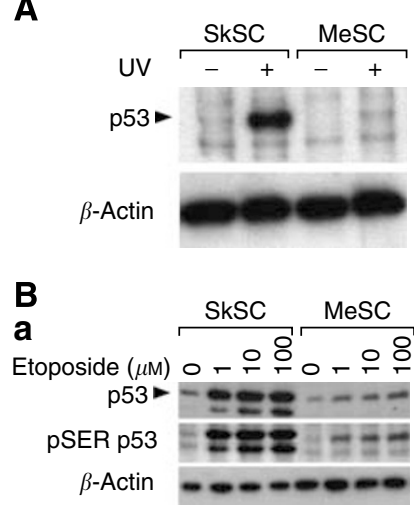

b

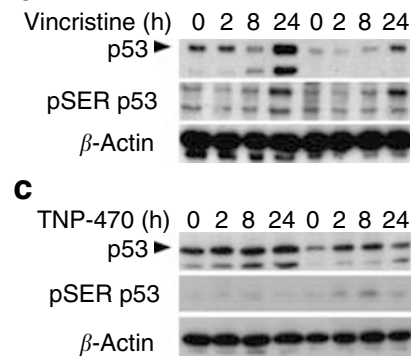

c

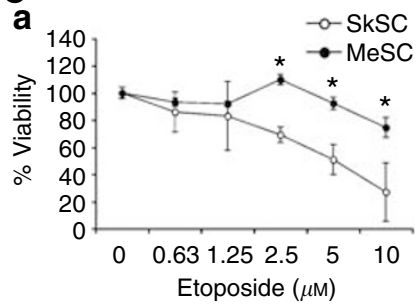

b
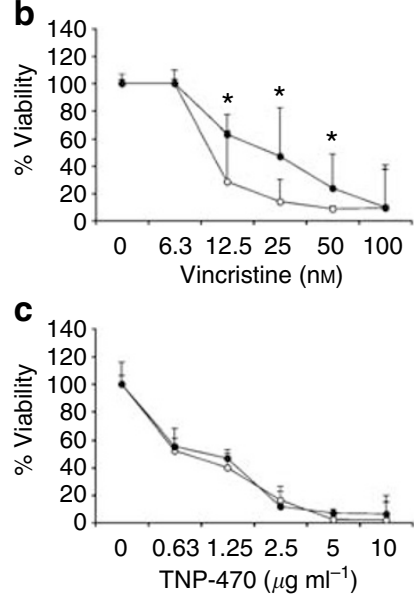

Figure 2 p53 function in tumour stromal cells. (A) Western blotting of whole-cell lysates from UV-treated SkSC and MeSC. Cells were treated with $100 \mathrm{~mJ} \mathrm{~cm}^{-2}$ for $5 \mathrm{~min}$ and lysates were prepared $6 \mathrm{~h}$ later. (B) Western blotting of whole-cell lysates from $8 \mathrm{~h}$ etoposide-treated (a), I nM vincristine-treated (b), or $10 \mu \mathrm{g} \mathrm{ml}^{-1}$ TNP-470-treated cells (c). The same blots were stripped and re-probed with rabbit polyclonal pSERI5 p53 antibodies and then mouse monoclonal $\beta$-actin antibodies. (C) Doseresponse curves for etoposide (a), vincristine (b), and TNP-470 (c). Cells were plated in triplicate and treated with each drug for $72 \mathrm{~h}$ before dispersing in trypsin and counting. *Results are statistically significant $(P<0.05)$ by student's $t$-test.

were used to amplify a $1173 \mathrm{bp}$ fragment that was subcloned into the PCR-2 vector (Invitrogen, Carlsbad, CA, USA). Plasmid DNA was cloned in Top10 cells, purified, and sequenced using the same primers for PCR amplification. Sequences were analysed using Chromas software.

\section{Karyotyping}

Karyotyping was carried out by the Brigham and Women's Cytogenetics Core Facility, Boston, MA, USA.

\section{siRNA}

Mouse siRNA for p53 was purchased from Dharmacon (Lafayette, CO, USA). Cells were transfected using Silentfect transfection reagent according to the manufacturer's instructions (BioRad, Hercules, CA, USA).

\section{RESULTS}

\section{Stromal cell characterisation}

Previously, our laboratory identified cytogenetic alterations in tumour-specific endothelial cells (Hida et al, 2004). In the present study, we found that isolates of endothelial cells from A375 melanoma were frequently overtaken by fibroblast-like stromal cells (MeSC), which grew on average two to three times the rate of normal SC from mouse skin (SkSC) (Figure 1A). In contrast to tumour endothelial cells described by Hida et al (2004), these cells

A

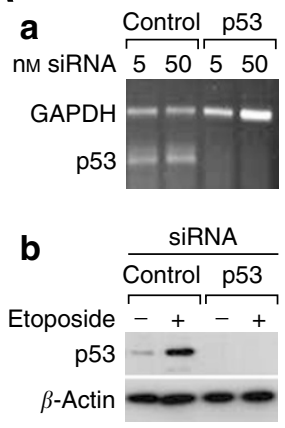

B
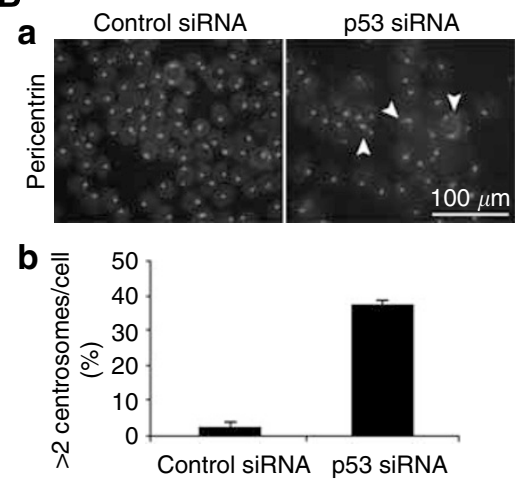

C
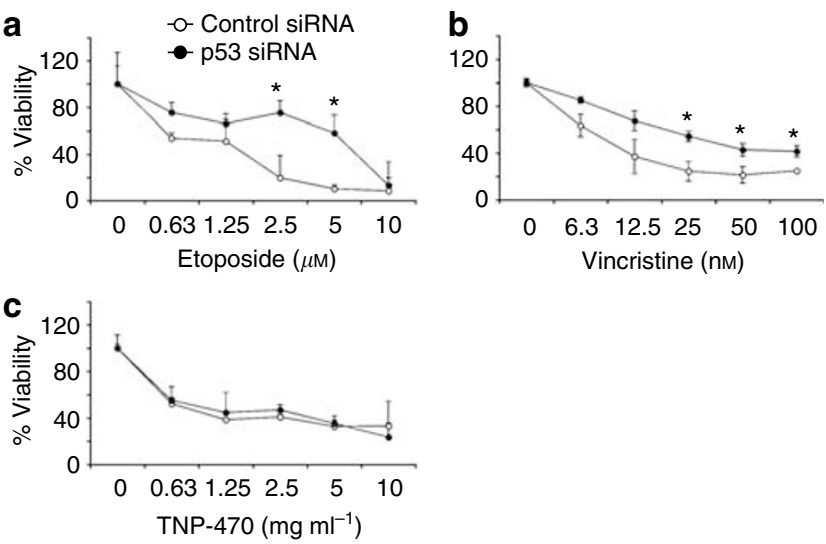

Figure 3 p53 knockdown in SkSC decreases sensitivity to etoposide and vincristine. (A) p53 knockdown was complete at the RNA (a) and protein levels (b) using siRNA. (B) p53 knockdown resulted in multiple lessuniform centrosomes (a), and about $30 \%$ of the cells had an abnormal $>2$ pericentrin signals per cell (b). (C) Cell numbers determined in p53 knockdown SkSC after a $72 \mathrm{~h}$ treatment with etoposide $(\mathbf{a})$, vincristine $(\mathbf{b})$, and TNP-470 (c). Cells were plated in triplicate and treated with each drug for $72 \mathrm{~h}$ before dispersing in trypsin and counting. *Results are statistically significant $(P<0.05)$ by student's $t$-test.

were negative for the EC markers CD31 and VEGFR2 (data not shown), but positive for caldesmon, CD105, and FSP-1 (Figure 1B). $\alpha$-Smooth muscle actin expression was restricted to the SC from melanoma, possibly owing to their 'activated' or myofibroblastlike phenotype observed in most tumours (Orimo et al, 2005). On the basis of morphology and marker expression, the isolated SC used in this study were identified as fibroblasts or myofibroblasts.

\section{p53 function in normal and tumour stromal cells}

Ultraviolet (UV) light causes DNA damage, which is repaired via a p53-dependent mechanism (Nelson and Kastan, 1994). As expected, p53 levels were increased in normal SkSC after UV treatment (Figure 2A). Strikingly, p53 only marginally accumulated in MeSC after treatment with UV light. Next, the p53 responses in SkSC or MeSC following treatment with different p53activating drugs were determined. Although etoposide caused an approximate five-fold increase in p53 and SER15-phosphorylated p53 in SkSC at all doses, only a two-fold increase in p53 was evident in MeSC (Figure 2B). Time course experiments using vincristine produced similar results, with a maximum seven- to eightfold increase in p53 levels in SkSC after drug treatment and only a one- to twofold increase in MeSC. TNP-470, a compound not known as a p53 inducer, did not cause a remarkable upregulation of $\mathrm{p} 53$ or $\mathrm{p} 53$ phosphorylation at any time point in both SC. 
A

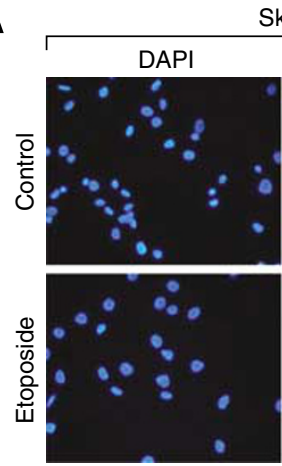

B

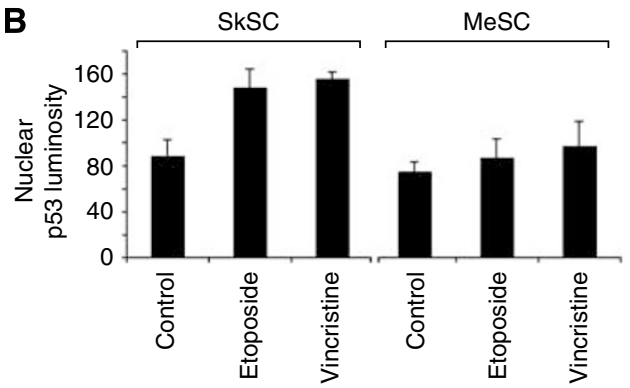

D

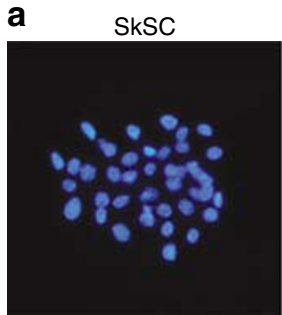

SkSC

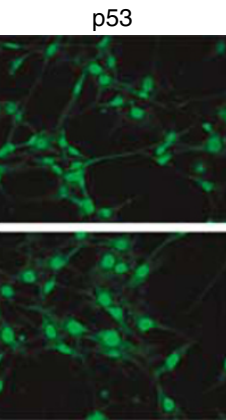

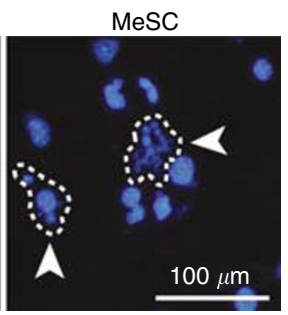

MeSC

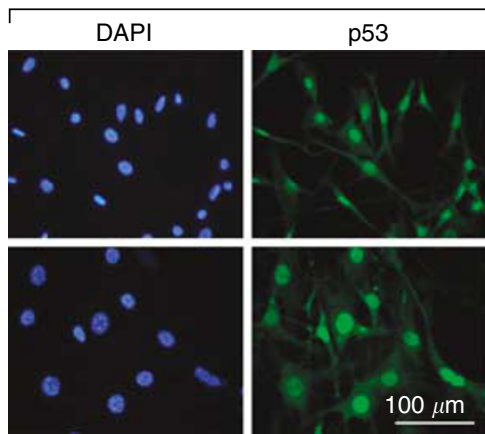

C

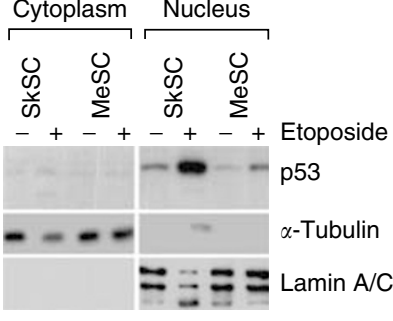

b

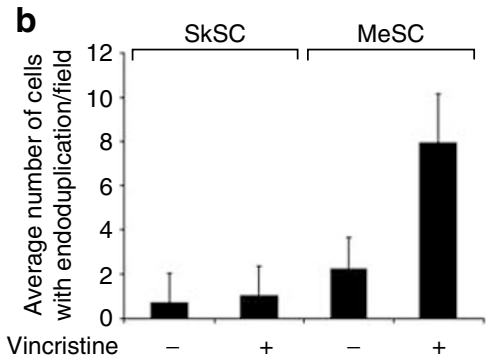

Figure 4 p53 localises to the nucleus in SkSC and MeSC, but the total cellular p53 pool is reduced. (A) p53 immunofluorescence in SkSC and MeSC treated with etoposide ( $10 \mu \mathrm{M}, 8 \mathrm{~h}$ ). Note the predominant nuclear staining for p53 in both the cell types, although the p53 signal in MeSC is diminished. (B) The luminosity for the nuclear $\mathrm{p} 53$ signal in treated and untreated cells was measured in 10 cells from three random fields and plotted. (C) Western blots of purified nuclear and cytoplasmic fractions from etoposide-treated cells ( $10 \mu \mathrm{M}, 8 \mathrm{~h}$ ). As loading controls, blots were stripped and re-probed with $\gamma$-tubulin (cytoplasm) and lamin A/C (nucleus). (D) Vincristine-treated cells (I nM, 24h) were methanol-fixed, and the nuclei stained with DAPI. The circled cells are single cells, and the arrows point to endoduplicated nuclei (a). Cells in 10 random fields were counted, and the average number of cells with multiple nuclei ( $>3$ per cell) was plotted $(\mathbf{b})$.

\section{MeSC are less sensitive to p53-activating drugs}

We compared the viability of SkSC and MeSC following treatment with each p53-activating drug. Although both cell types were growthinhibited by etoposide and vincristine, MeSC were consistently less sensitive when compared to SkSC. In contrast, no difference in viability was seen in SkSC or MeSC treated with TNP-470, which also did not upregulate p53 (Figure 2C). To confirm a p53-specific effect, we knocked down p53 in SkSC using p53 siRNA. p53 knockdown was complete at the RNA and protein levels (Figure 3A). As a readout of $\mathrm{p} 53$ function, we measured centrosome numbers in SkSC after p53 knockdown (Bennett et al, 2004). p53 knockdown in SkSC resulted in multiple centrosomes per cell, consistent with the role of p53 in regulating centrosome duplication (Figure 3B). Furthermore, the dose-response curves for etoposide and vincristine showed that p53 knockdown rendered SkSC less sensitive to each drug (Figure 3C). No change in sensitivity to TNP-470 was observed, consistent with the failure of TNP-470 to activate p53.

\section{p53 localises to the nucleus in MeSC but the total cellular p53 pool is reduced}

As p53 mutations may result in its sequestration in cytoplasm or nucleus, we determined the subcellular localisation of p53 by immunofluorescence and cell fractionation studies. By immunofluorescence, p53 localised predominately to the nucleus in both SkSC and MeSC in untreated cells and in cells treated with etoposide (Figure 4A). However, when the results were quantified, nuclear p53 levels in SkSC treated with etoposide or vincristine were approximately twofold higher compared to MeSC (Figure 4B). Western blotting of purified nuclear and cytosolic extracts of etoposide-treated cells confirmed that p53 was predominately localised to the nucleus, where it was strikingly increased in SkSC, but not MeSC (Figure 4C). It is worth noting that almost 10 times as many cells with endoduplicated nuclei (nuclear division without cell division) were detected in MeSC compared to SkSC after vincristine treatment (Figure 4D). Endoduplication is commonly observed in p53-null mouse embryonic fibroblasts treated with microtubule inhibitors and occurs due to checkpoint failure in the absence of normal p53 function (Lanni and Jacks, 1998).

\section{MeSC fail to arrest in $\mathrm{G}_{2}$ after etoposide treatment}

As the growth inhibitory effects of etoposide are mainly due to $\mathrm{G}_{2}$ arrest, cell cycle status in etoposide-treated cells was determined by propidium iodide (PI) staining followed by FACS. In SkSC 
122
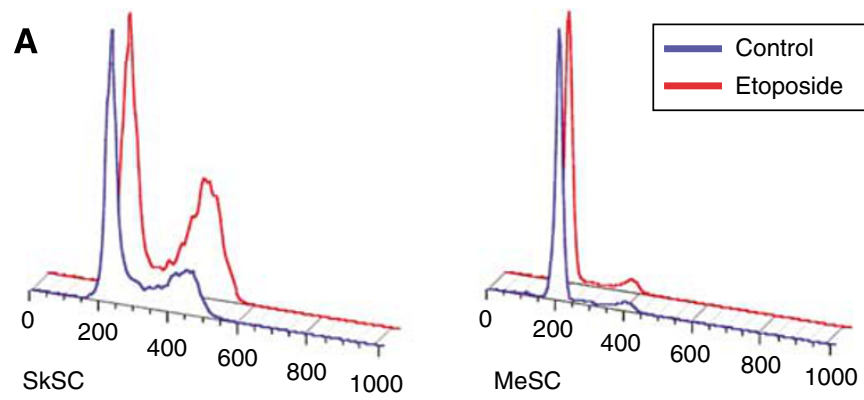

B

\begin{tabular}{|l|cc|cc|}
\hline \multirow{2}{*}{} & \multicolumn{2}{|c|}{ SkSC } & \multicolumn{2}{c|}{ MeSC } \\
\cline { 2 - 5 } & Control & Etoposide & Control & Etoposide \\
\hline $\mathrm{G}_{0} \mathrm{G}_{1}$ & 68.6 & 67.45 & 88.65 & 88.0 \\
$\mathrm{~S}$ & 14.1 & 8.64 & 5.28 & 6.27 \\
$\mathrm{G}_{2} \mathrm{M}$ & 12.45 & 20.2 & 5.0 & 5.55 \\
\hline
\end{tabular}

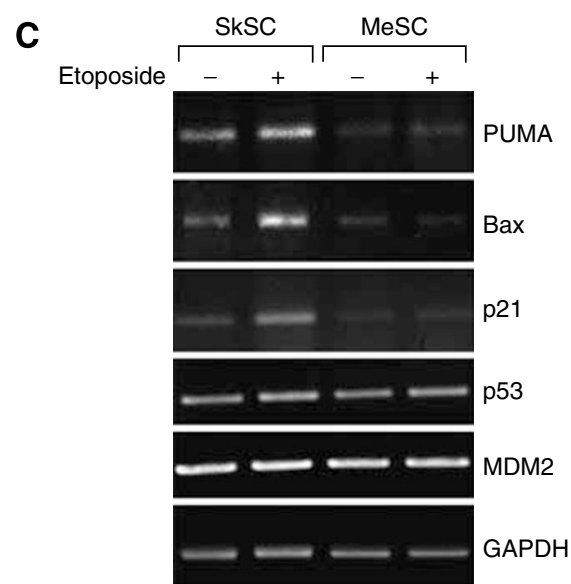

Figure 5 MeSC fail to arrest in $\mathrm{G}_{2}$ after etoposide treatment. (A) Cells treated with I $\mu \mathrm{M}$ etoposide were analysed by FACS $24 \mathrm{~h}$ later. Note the larger $G_{2}$ peak on the histogram in etoposide-treated SkSC but not in MeSC. (B) DNA histograms were analysed and the results from two experiments were averaged and are shown in the figure (numbers are per cent). (C) Semiquantitative RT-PCR analysis for p53-indicible genes in SC treated with I $\mu \mathrm{M}$ etoposide for $24 \mathrm{~h}$.

treated with etoposide, the number of cells in $\mathrm{G}_{2}$ almost doubled compared to untreated cells. In contrast, MeSC failed to accumulate in $\mathrm{G}_{2}$ (Figure $5 \mathrm{~A}$ and $\mathrm{B}$ ). By semiquantitative RT-PCR, the expression of p53-inducible genes related to cell cycle arrest and apoptosis including PUMA, Bax, and p21 was upregulated in SkSC, but not in MeSC after etoposide treatment (Figure 5C). Notably, the constitutive levels of these genes were also qualitatively lower in MeSC relative to SkSC. No differences in p53 or in the negative regulator of p53 stability, MDM2, were detected. Taken together, these results were consistent with a failed induction of $\mathrm{p} 53$ and p53-inducible genes related to apoptosis or cell cycle arrest in MeSC after etoposide treatment.

\section{MeSC fail to undergo apoptosis after vincristine treatment}

Dual staining using PI and annexin V (AV) in vincristine-treated cells indicated an increase in $\mathrm{AV}^{+} / \mathrm{PI}^{+}$cells in SkSC, but not MeSC (Figure 6A). The number of early apoptotic $\mathrm{AV}^{+}$cells increased six times above untreated cells in SkSC but only two times in MeSC (Figure 6B). Thus, the decreased sensitivity to vincristine in MeSC relative to SkSC was most likely due to decreased p53-dependent apoptosis.
A
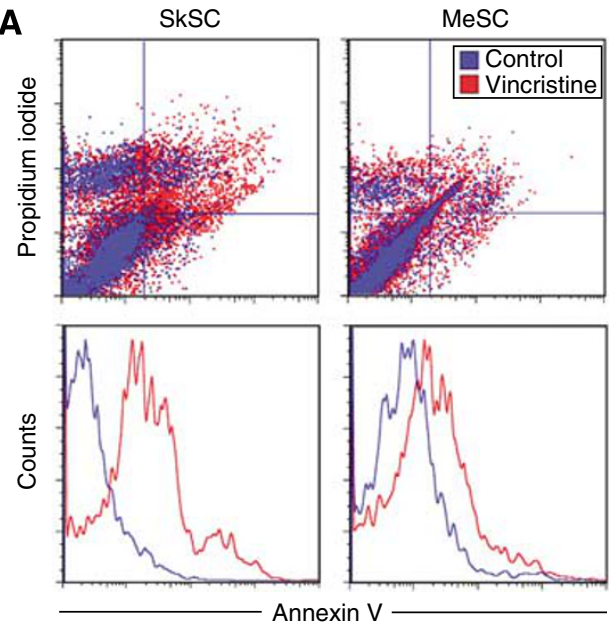

$\mathbf{B}$

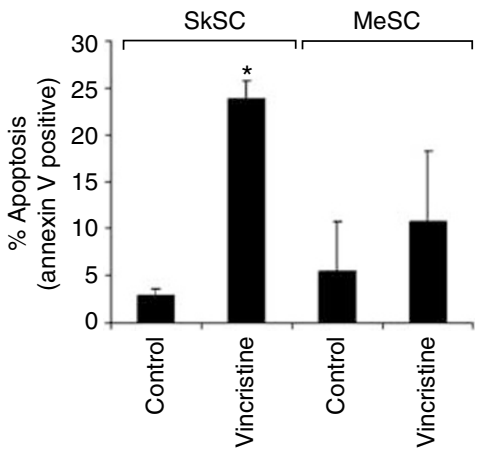

Figure 6 MeSC fail to undergo apoptosis after vincristine treatment. (A) Cells treated with I nM vincristine for $24 \mathrm{~h}$ were double stained with $\mathrm{Pl}$ and $\mathrm{AV}$ and analysed by FACS. An increase in $\mathrm{Pl}^{+} / \mathrm{AV}^{+}$and $\mathrm{PI}^{-} / \mathrm{AV}^{+}$cells was detected in vincristine-treated SkSC compared to MeSC. (B) The average numbers of early apoptotic $\mathrm{AV}^{+}$cells from two experiments were plotted. *Results are statistically significant $(P<0.05)$ by student's $t$-test.

\section{p53 function is impaired in stromal cells from PC3 and TRAMP prostate tumours}

To determine if SC from different tumours also showed diminished p53 function, we isolated SC from PC3 xenografts and spontaneous prostate tumours in TRAMP mice. Both PC3SC and TRAMPSC had a fibroblast-like morphology, were negative for EC markers (data not shown), and were positive for FSP-1, indicating that they were tumour-associated fibroblasts, similar to SC from A375 melanoma (Figure 7A). For all tumour xenografts used in this study, diphtheria toxin was used to eliminate human tumour cells from cultures (Arbiser et al, 1999). Diphtheria toxin could not be used in the TRAMP model because these are spontaneous tumours with no human component. However, TRAMPSC did not express epithelial-specific E-cadherin by FACS or immunofluorescence, indicating absence of contaminating tumour cells (data not shown). Similar to MeSC, both PC3SC and TRAMPSC showed only modest increases in p53 after treatment with etoposide or vincristine, whereas p53 levels in SkSC were markedly upregulated (Figure 7B). Both vincristine and etoposide treatment increased SER 15 phosphorylation in both normal and tumour SC, although the levels were reduced in tumour SC presumably due to the decrease in total p53 levels. However, although SER20 was phosphorylated in normal SC after vincristine treatment, no increase in SER20 phosphorylation was evident in tumour SC. No SER20 phosphorylation was detected in SkSC or PC3SC after treatment with etoposide (data not shown). Similar to MeSC, and in good accord with the diminished p53 levels, TRAMPSC and PC3SC were less 
A

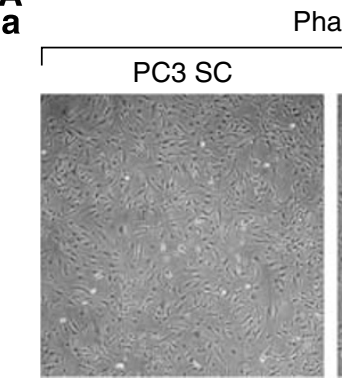

Phase

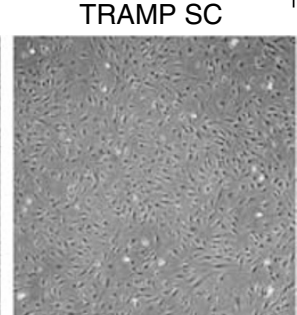

b

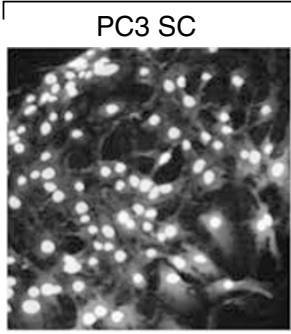

FSP-1 TRAMP SC

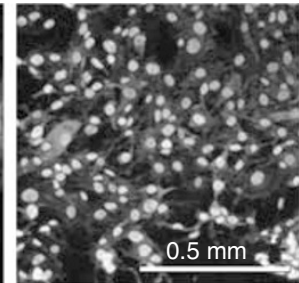

B
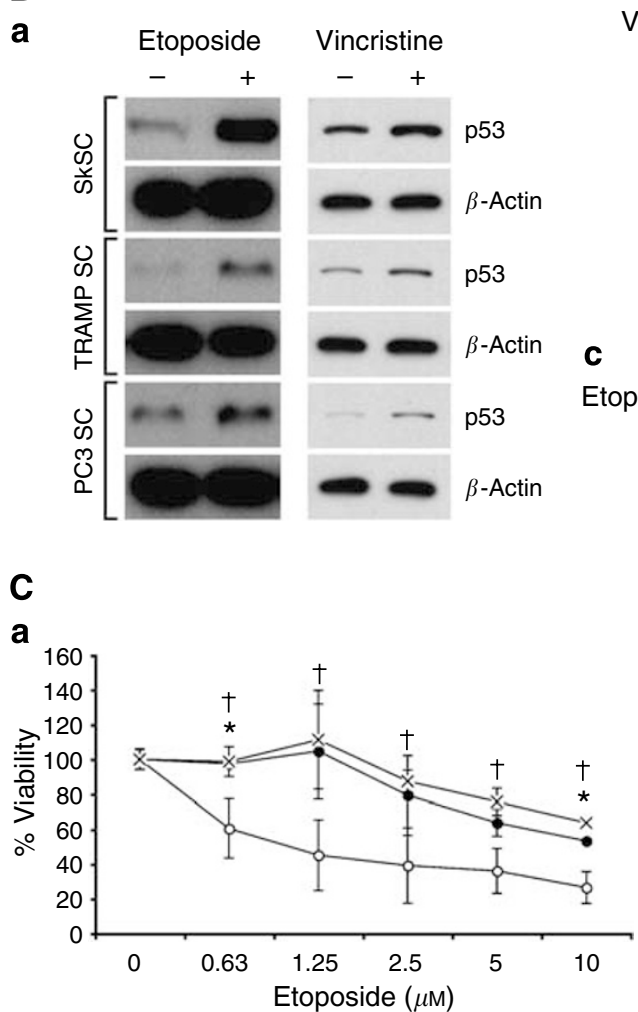

b

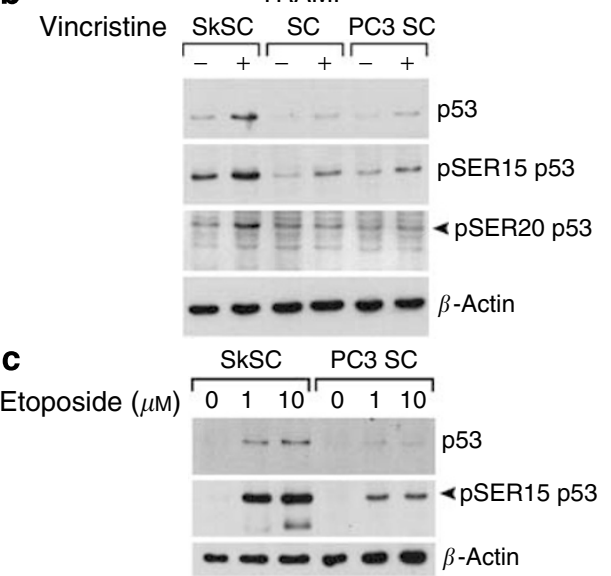

Figure 7 p53 function is impaired in stromal cells from PC3 and TRAMP prostate tumours. (A) Both PC3SC and TRAMPSC had a fibroblast-like morphology $(\mathbf{a})$ and uniformly expressed FSP-I (b). (B) After treatment with etoposide $(10 \mu \mathrm{M}, 8 \mathrm{~h})$ or vincristine (I nM, $24 \mathrm{~h})$, p53 accumulation was diminished in TRAMPSC and PC3SC compared to normal SkSC (a). Western blotting for pSERI 5 and pSER20 after vincristine (b) (I nM, $24 \mathrm{~h}$ ) or etoposide $(8 \mathrm{~h})$ treatment $(\mathbf{c})$. Blots were striped and re-probed with p53 or $\beta$-actin antibodies. (C) Viabilities of TRAMPSC and PC3SC after treatment with etoposide (a) or vincristine (b). Cells were plated in triplicate and treated with each drug for $72 \mathrm{~h}$ before dispersing in trypsin and counting. Asterisk (*) indicates results are statistically significant $(P<0.05)$ by Student's t-test when comparing SkSC vs PC3 SC and a dagger $(\dagger)$ when comparing SkSC vs TRAMP SC.

sensitive to etoposide and vincristine compared to SkSC (Figure 7C). These results suggest that tumour-associated SC from different tumour types show diminished p53 protein levels and impaired p53 function.

\section{Tumour stromal cells have multiple centrosomes and aneuploid karyotypes}

Hida et al (2004) recently reported abnormal centrosomes and abnormal karyotypes in tumour-specific endothelial cells. About $30 \%$ of MeSC, TRAMPSC, and PC3SC also had abnormal multiple centrosomes by pericentrin staining (Figure 8A). Karyotypes on the isolated cells showed that all tumour SC had heterogeneous aneuploid chromosomes whereas SkSC were normal (Figure 8B). Taken together, SC from different types of tumours (melanoma $v s$ prostate) and different models (xenograft $v s$ spontaneous) are characterised by aneuploid karyotypes and multiple centrosomes.

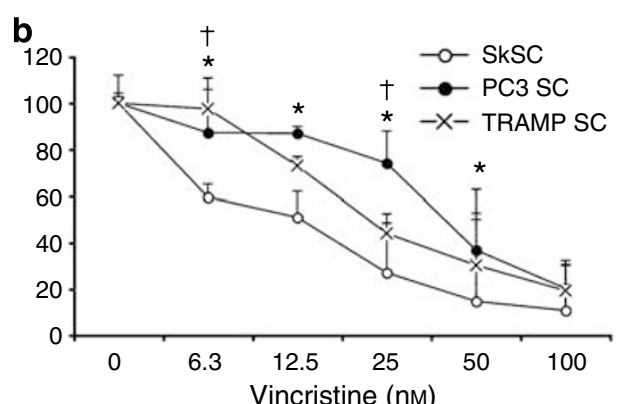

\section{DISCUSSION}

Our study provides evidence for impaired p53 function in tumourassociated SC. All tumour SC examined were characterised by diminished p53 protein levels, decreased sensitivity to cytotoxic drugs, and genomic instability indicated by multiple centrosomes and aneuploid karyotypes. Although the nature of the p53 defect in tumour SC is not yet clear, impaired p53 function in tumour stroma could enable SC survival in the tumour microenvironment and contribute to genomic instability.

Host SC can constitute a significant percentage of the total tumour bulk as shown in GFP-SCID mice implanted with tumour xenografts (Udagawa et al, 2006). Tumour-associated fibroblasts or mesenchymal-like cells comprise a large portion of the tumour stroma. Collectively, these cells might arise from tissue-resident activated fibroblasts, tumour epithelial cells undergoing epithelialto-mesenchymal transition, resident stem or mesenchymal-like cells, or bone marrow-derived progenitors. Recently, mesenchymal 


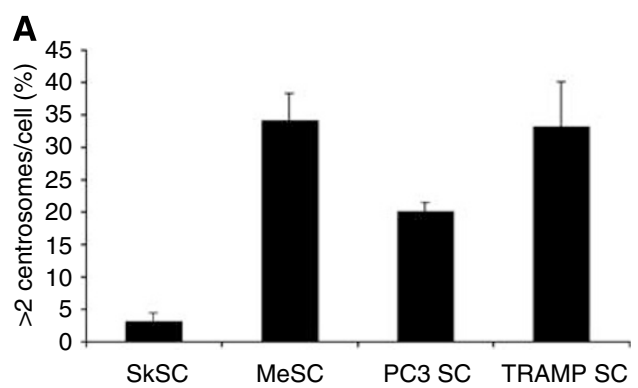

B

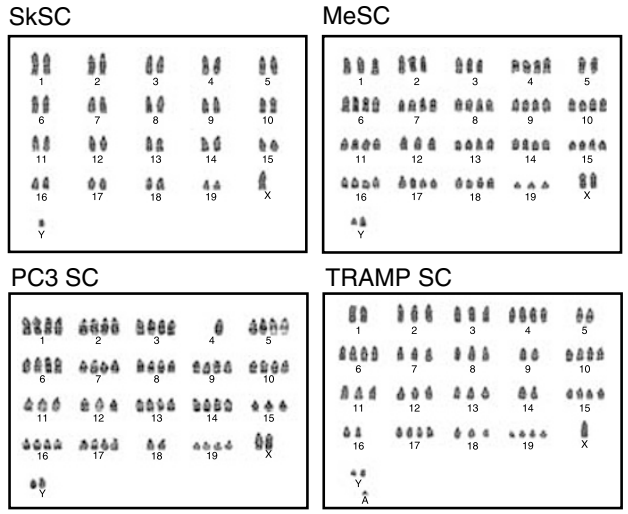

Figure 8 Tumour stromal cells have multiple centrosomes and aneuploid karyotypes. (A) Centrosomes were labeled with pericentrin antibodies and counted. One hundred cells were scored and averaged from three different fields. (B) Karyotypes for SkSC, MeSC, PC3SC, and TRAMPSC showing aneuploidy in all tumour SC, whereas SkSC were normal.

stem cells localised to breast carcinoma were shown to increase metastatic potency (Karnoub et al, 2007). Thus, by providing scaffolds for tumour cells and other SC, and by producing growth factors and chemotactic factors for inflammatory cells and vascular progenitors, tumour SC can enable tumour growth and possibly metastasis (Olumi et al, 1999; Bhowmick et al, 2004). Targeting tumour SC may therefore be a viable approach for eliminating solid tumours (Hofmeister et al, 2008).

Recently, our laboratory reported that endothelial cells from human-to-mouse xenografts were aneuploid with centrosome abnormalities 4-6 weeks after implantation (Hida et al, 2004). Additional studies have confirmed both cytogenetic and epigenetic alterations in tumour-associated SC and tissue adjacent to carcinoma (Deng et al, 1994; Moinfar et al, 2000; Streubel et al, 2004). The mechanism(s) of these cytogenetic changes in tumour stroma are not yet clear. One possibility is that SC and tumour cells may fuse, as hypothesised in cases of allelic imbalance and genomic instability in human breast stroma (Weber et al, 2006). However, we found no evidence of human DNA in the karyotypes of murine tumour SC from human-to-mouse xenografts. Selection pressure for SC with diminished p53 function, and thus a survival advantage in the tumour microenvironment, is also a possibility. For example, nonautonomous oncogenic stress in tumour epithelium may result in the selection of SC with $\mathrm{LOH}$ at the p53 locus (Lu et al, 2001; Hill et al, 2005; Kiaris et al, 2005). It is likely that impaired p53 function in tumour SC might enable the propagation of cells with damaged DNA due to checkpoint failure leading to chromosomal instability.

A potential caveat related to targeting tumour SC is the loss of function of tumour suppressor genes in the stromal compartment (Patocs et al, 2007). Hill et al (2005) described widespread p53 loss in tumour mesenchyme after 20-25 weeks in a prostate cancer model. We evaluated p53 function and performed karyotypes in TRAMPSC at 22 weeks following tumour initiation and in SC from tumour xenografts at 4-6 weeks postimplantation. In either case, alterations in p53 function, diminished p53 protein levels and abnormal karyotypes were evident, irrespective of time or tumour model. At present, we have been unable to detect mutations in the p53-coding regions in SC, nor could we detect p53 LOH by real-time-PCR using genomic DNA (data not shown). Although real-time-PCR is a sensitive and well-established method to detect $\mathrm{LOH}$, heterogeneity in p53 status in the cultured tumour SC could confound these results. Therefore, $\mathrm{LOH}$ may only be evident on a cell-to-cell basis. Further studies will be needed to address the mechanism of impaired p53 function in tumour SC in our in vitro system and whether tumour SC can acquire drug resistance in vivo.

It is also possible that alterations in p53-interacting proteins including the kinases responsible for p53 phosphorylation could contribute to impaired p53 function in tumour SC. For example, DNA damage imparts a well-characterised phosphorylation of SER15 and SER20 in the p53 transcriptional activation domain. Phosphorylation of these residues is thought to inhibit the interaction of p53 with MDM2 and increase its stability (Appella and Anderson, 2001). Although lower levels of p53 SER15 phosphorylation were evident in tumour SC in this study, this was most likely due to the decrease in total levels of the p53 protein. On the other hand, SER20 did not appear to be phosphorylated in tumour SC relative to normal SC after vincristine treatment. It remains possible that in tumour SC, alterations in pathways secondary to the stability of p53, rather than direct alterations in p53, could impart a destabilising effect on p53 leading to its degradation.

The idea that tumour SC can contribute to tumour growth and perhaps metastasis is an emerging concept in cancer biology (Karnoub et al, 2007). Though alterations in p53 in tumour SC have been shown previously in vivo, we show that diminished p53 function accompanies genomic instability and decreased sensitivity to cytotoxic drugs.

\section{ACKNOWLEDGEMENTS}

This work was supported by NIH grants CA37392 and CA45548. ACD wishes to thank Leonora DeBella and the American Cancer Society for supporting his research with a postdoctoral fellowship. We thank Drs Akiko Mammoto and Elisa Boscolo for the lamin A/C and tubulin antibodies. We thank Dr Dipak Panigrahy for the TNP-470. We thank Kristin Johnson for her excellent assistance in preparing the figures for this manuscript and the MRDDRC for the DNA sequencing.

\section{REFERENCES}

Allinen M, Beroukhim R, Cai L, Brennan C, Lahti-Domenici J, Huang H, Porter D, Hu M, Chin L, Richardson A, Schnitt S, Sellers WR, Polyak K (2004) Molecular characterization of the tumor microenvironment in breast cancer. Cancer Cell 6: 17-32

Appella E, Anderson CW (2001) Post-translational modifications and activation of p53 by genotoxic stresses. Eur J Biochem 268: 2764-2772

Arbiser JL, Raab G, Rohan RM, Paul S, Hirschi K, Flynn E, Price ER, Fisher DE, Cohen C, Klagsbrun M (1999) Isolation of mouse stromal cells associated with a human tumor using differential diphtheria toxin sensitivity. Am J Pathol 155: 723-729

Bennett RA, Izumi H, Fukasawa K (2004) Induction of centrosome amplification and chromosome instability in p53-null cells by transient 
exposure to subtoxic levels of S-phase-targeting anticancer drugs. Oncogene 23: 6823-6829

Bhowmick NA, Neilson EG, Moses HL (2004) Stromal fibroblasts in cancer initiation and progression. Nature 432: $332-337$

Bunz F, Hwang PM, Torrance C, Waldman T, Zhang Y, Dillehay L, Williams J, Lengauer C, Kinzler KW, Vogelstein B (1999) Disruption of p53 in human cancer cells alters the responses to therapeutic agents. J Clin Invest 104: 263 - 269

Burdelya LG, Komarova EA, Hill JE, Browder T, Tararova ND, Mavrakis L, Dicorleto PE, Folkman J, Gudkov AV (2006) Inhibition of p53 response in tumor stroma improves efficacy of anticancer treatment by increasing antiangiogenic effects of chemotherapy and radiotherapy in mice. Cancer Res 66: $9356-9361$

Coussens LM, Werb Z (2002) Inflammation and cancer. Nature 420: $860-867$

Deng G, Chen LC, Schott DR, Thor A, Bhargava V, Ljung BM, Chew K, Smith HS (1994) Loss of heterozygosity and p53 gene mutations in breast cancer. Cancer Res 54: 499-505

Dvorak HF (1986) Tumors: wounds that do not heal. Similarities between tumor stroma generation and wound healing. N Engl J Med 315: $1650-1659$

Fukino K, Shen L, Matsumoto S, Morrison CD, Mutter GL, Eng C (2004) Combined total genome loss of heterozygosity scan of breast cancer stroma and epithelium reveals multiplicity of stromal targets. Cancer Res 64: $7231-7236$

Gorgoulis VG, Vassiliou LV, Karakaidos P, Zacharatos P, Kotsinas A, Liloglou T, Venere M, Ditullio Jr RA, Kastrinakis NG, Levy B, Kletsas D, Yoneta A, Herlyn M, Kittas C, Halazonetis TD (2005) Activation of the DNA damage checkpoint and genomic instability in human precancerous lesions. Nature 434: $907-913$

Hida K, Hida Y, Amin DN, Flint AF, Panigrahy D, Morton CC, Klagsbrun M (2004) Tumor-associated endothelial cells with cytogenetic abnormalities. Cancer Res 64: 8249-8255

Hill R, Song Y, Cardiff RD, Van Dyke T (2005) Selective evolution of stromal mesenchyme with p53 loss in response to epithelial tumorigenesis. Cell 123: $1001-1011$

Hinz B, Phan SH, Thannickal VJ, Galli A, Bochaton-Piallat ML, Gabbiani G (2007) The myofibroblast: one function, multiple origins. Am J Pathol 170: $1807-1816$

Hofmeister V, Schrama D, Becker JC (2008) Anti-cancer therapies targeting the tumor stroma. Cancer Immunol Immunother 57: 1-17

Kaplan RN, Riba RD, Zacharoulis S, Bramley AH, Vincent L, Costa C, MacDonald DD, Jin DK, Shido K, Kerns SA, Zhu Z, Hicklin D, Wu Y, Port JL, Altorki N, Port ER, Ruggero D, Shmelkov SV, Jensen KK, Rafii S, Lyden D (2005) VEGFR1-positive haematopoietic bone marrow progenitors initiate the pre-metastatic niche. Nature 438: 820-827

Karnoub AE, Dash AB, Vo AP, Sullivan A, Brooks MW, Bell GW, Richardson AL, Polyak K, Tubo R, Weinberg RA (2007) Mesenchymal stem cells within tumour stroma promote breast cancer metastasis. Nature 449: $557-563$

Kiaris H, Chatzistamou I, Trimis G, Frangou-Plemmenou M, Pafiti-Kondi A, Kalofoutis A (2005) Evidence for nonautonomous effect of p53 tumor suppressor in carcinogenesis. Cancer Res 65: 1627-1630

Kurose K, Gilley K, Matsumoto S, Watson PH, Zhou XP, Eng C (2002) Frequent somatic mutations in PTEN and Tp53 are mutually exclusive in the stroma of breast carcinomas. Nat Genet 32: 355-357
Lanni JS, Jacks T (1998) Characterization of the p53-dependent postmitotic checkpoint following spindle disruption. Mol Cell Biol 18: $1055-1064$

Liotta LA, Kohn EC (2001) The microenvironment of the tumour-host interface. Nature 411: 375-379

Lu X, Magrane G, Yin C, Louis DN, Gray J, Van Dyke T (2001) Selective inactivation of p53 facilitates mouse epithelial tumor progression without chromosomal instability. Mol Cell Biol 21: 6017-6030

Martins CP, Brown-Swigart L, Evan GI (2006) Modeling the therapeutic efficacy of p53 restoration in tumors. Cell 127: 1323-1334

Moinfar F, Man YG, Arnould L, Bratthauer GL, Ratschek M, Tavassoli FA (2000) Concurrent and independent genetic alterations in the stromal and epithelial cells of mammary carcinoma: implications for tumorigenesis. Cancer Res 60: 2562-2566

Nelson WG, Kastan MB (1994) DNA strand breaks: the DNA template alterations that trigger p53-dependent DNA damage response pathways. Mol Cell Biol 14: 1815-1823

Olumi AF, Grossfeld GD, Hayward SW, Carroll PR, Tlsty TD, Cunha GR (1999) Carcinoma-associated fibroblasts direct tumor progression of initiated human prostatic epithelium. Cancer Res 59: 5002-5011

Orimo A, Gupta PB, Sgroi DC, Arenzana-Seisdedos F, Delaunay T, Naeem R, Carey VJ, Richardson AL, Weinberg RA (2005) Stromal fibroblasts present in invasive human breast carcinomas promote tumor growth and angiogenesis through elevated SDF-1/CXCL12 secretion. Cell 121: $335-348$

Patocs A, Zhang L, Xu Y, Weber F, Caldes T, Mutter GL, Platzer P, Eng C (2007) Breast-cancer stromal cells with Tp53 mutations and nodal metastases. N Engl J Med 357: 2543 -2551

Pelham RJ, Rodgers L, Hall I, Lucito R, Nguyen KC, Navin N, Hicks J, Mu D, Powers S, Wigler M, Botstein D (2006) Identification of alterations in DNA copy number in host stromal cells during tumor progression. Proc Natl Acad Sci USA 103: 19848 - 19853

Sappino AP, Skalli O, Jackson B, Schurch W, Gabbiani G (1988) Smoothmuscle differentiation in stromal cells of malignant and non-malignant breast tissues. Int I Cancer 41: 707-712

Seemayer TA, Lagace R, Schurch W, Tremblay G (1979) Myofibroblasts in the stroma of invasive and metastatic carcinoma: a possible host response to neoplasia. Am J Surg Pathol 3: 525-533

Streubel B, Chott A, Huber D, Exner M, Jager U, Wagner O, Schwarzinger I (2004) Lymphoma-specific genetic aberrations in microvascular endothelial cells in B-cell lymphomas. $N$ Engl J Med 351: 250-259

Tlsty TD (2001) Stromal cells can contribute oncogenic signals. Semin Cancer Biol 11: 97-104

Udagawa T, Puder M, Wood M, Schaefer BC, D’Amato RJ (2006) Analysis of tumor-associated stromal cells using SCID GFP transgenic mice: contribution of local and bone marrow-derived host cells. FASEB J 20: 95- 102

Ventura A, Kirsch DG, McLaughlin ME, Tuveson DA, Grimm J, Lintault L, Newman J, Reczek EE, Weissleder R, Jacks T (2007) Restoration of $\mathrm{p} 53$ function leads to tumour regression in vivo. Nature 445: $661-665$

Weber F, Shen L, Fukino K, Patocs A, Mutter GL, Caldes T, Eng C (2006) Total-genome analysis of BRCA1/2-related invasive carcinomas of the breast identifies tumor stroma as potential landscaper for neoplastic initiation. Am J Hum Genet 78: 961 -972 